\title{
Quercetin LipoMicel-A Novel Delivery System to Enhance Bioavailability of Quercetin
}

Julia Solnier(D, $\mathrm{PHD}^{1}{ }^{1}{ }^{*}$, Chuck Chang, Bsc ${ }^{1}$, Kyle Roh, BSc ${ }^{1}$, Min Du, MSc ${ }^{1}$, Yun Chai Kuo, BEng ${ }^{1}$, Mary Hardy, $\mathrm{MD}^{2}$, Michael Lyon, $\mathrm{MD}^{3,4}$, Roland Gahler ${ }^{5}$

${ }^{1}$ ISURA, Burnaby, BC, Canada

${ }^{2}$ Association of Integrative and Holistic Medicine, San Diego, California, USA

${ }^{3}$ Obesity Medicine and Diabetes Institute, Coquitlam, BC, Canada

${ }^{4}$ Department of Family Practice, Faculty of Medicine, University of British Columbia, Vancouver, BC, Canada

${ }^{5}$ Factors Group of Nutritional Products Inc. R\&D, Burnaby, BC, Canada

** Corresponding Author: jsolnier@factorsgroup.com

\begin{abstract}
BACKGROUND: Quercetin, a flavonoid found in plant-based foods, has a range of biological activities that may be beneficial for human health. The pharmacokinetic profile of quercetin remains, however, a limiting factor for its use as a nutritional supplement. Quercetin LipoMice ${ }^{\circledR}$ — a novel delivery system encapsulating quercetin into a liquid micelle matrix - has been designed to address the low bioavailability issue associated with non-conjugated forms by improving the absorption of quercetin.
\end{abstract}

OBJECTIVE: The purpose of this study was to evaluate the solubility and gastrointestinal absorption of quercetin in a novel Quercetin LipoMicel delivery system in healthy adult volunteers by comparing it with free quercetin and another commercial quercetin product. Several pharmacokinetic parameters were compared between these three formulations.

METHODS: Twelve healthy adult male and female volunteers aged between 21 and 65, with BMIs under 30, participated in a non-blinded, crossover bioavailability study conducted with three quercetin products. Each treatment contained a total dose of $500 \mathrm{mg}$ quercetin. Capillary whole blood samples from participants were collected serially at intervals from 0-24 hours. Quercetin concentrations were detected and measured by ultra-performance liquid chromatography (UHPLC) coupled to a Thermo QExactive Orbitrap Mass Spectrometer. Solubility of quercetin in water and simulated gastrointestinal media was determined by UHPLC.

RESULTS: Oral absorption of quercetin was significantly enhanced with the LipoMicel delivery system compared to free quercetin. Improvements in in vitro gastric stability and intestinal solubility were observed with LipoMicel, leading to significantly higher blood concentration and enhanced duration of a stable concentration of quercetin in the body. Compared to free quercetin, 8- and 9-fold increases in AUC and Cmax were attained with the LipoMicel delivery system, and 10-fold higher quercetin plasma concentrations detected at 12 hours after administration.

CONCLUSIONS: Quercetin LipoMicel represents an efficient delivery system for augmenting the bioavailability of quercetin in vivo. Significantly higher blood concentrations and a sustained release of quercetin over the study period was achieved following the administration of quercetin via the LipoMicel technology. Optimization in the in vivo bioavailability of quercetin may promote its salutary effects.

KEYWORDS: Anti-inflammation; antioxidative; antiviral; bioavailability; flavonoids; LipoMice ${ }^{\circledR}$; quercetin 


\section{Introduction}

Quercetin (2-(3,4-dihydroxyphenyl)-3,5,7-trihydroxychromen-4-one or 3,3',4',5,7-pentahydroxyflavone) is one of the most abundant dietary flavonoids and is widely distributed throughout the plant kingdom including fruits and vegetables such as apples, berries, capers, cilantro, onions, kale, green tea, and red wine [1]. It is present in several plants and foods including Ginkgo biloba, St. John's wort, American elder, and buckwheat tea. In its natural food matrix, quercetin exists in a bound form, e.g., attached to sugars, ethers, or phenolic acids, thus limiting its bioavailability (Wang et al., 2016). Purified quercetin appears as a yellow powder that is soluble in alcohol and lipophilic solvents but is poorly soluble in water [2].

Throughout the history of traditional or indigenous medicine, extracts from herbs and plants have been used to manage a variety of illnesses. The putative health promoting effects of quercetin have been reported as ranging across anti-allergic, anticancer, anti-inflammatory, antiviral, and cardiovascular protective activities [3, 4]. For example, the flavonol was found to reduce the release of inflammatory cytokines (e.g., IL-8) which may alleviate infection-related symptoms and possibly suppress inflammatory responses that are often associated with viral infections [5-7]. In the case of rheumatoid arthritis, quercetin has been reported to inhibit neutrophil activity, and reduce the plasma levels of inflammatory cytokines [8]. Kinker (2014) has suggested that the antioxidative potential of quercetin might prevent tissue damage by scavenging free radicals.

Even though quercetin may exert a broad range of biological activities, its poor pharmacokinetic profile strongly limits its clinical use.

Quercetin, orally administered, is subject to numerous degradation steps within the gastrointestinal system including: aggregate formation with salivary gland proteins in the mouth [9]; degradation to phenolic acids and limited absorption in the stomach at low $\mathrm{pH}$; glucuronidation, sulfation, and methylation at intestinal and hepatic level; and formation of an aglycone by enzymes of the microbiota $[10,11]$.

Moreover, the intake of quercetin from food may not be sufficient to achieve clinically significant blood concentrations because of the effects noted above. In addition, the intake of quercetin from the diet can be compromised by non-host related factors which may contribute to poor bioavailability of dietary flavonoids. For example, environmental factors such as storage, sun exposure, food processing factors like cooking or freezing, chemical structure (glycosylated or polymeric structure vs aglycone form) and the concentration in the dietary substrate [12] all have an impact on bioavailability. However, oxidation during food production and storage appeared to be the main cause of changes and degradation of quercetin [3].

Encapsulating bioactive compounds into water or liquid based matrices, may improve their chemical and gastrointestinal solubility. These carrier designs may further allow for better monitoring of the rate and site of action within the gastrointestinal tract by increasing the compound's absorption and subsequent metabolism. Enhanced delivery systems such as polymeric nanoparticles, structural modifications (glycosylation), liposomes, phytosomes, micelles, and emulsions have been suggested as strategies to improve gastrointestinal solubility and absorption [13]. A liquid micelle matrix composed of natural food grade ingredients was developed to potentially increase the bioavailability of quercetin.

The Quercetin LipoMicel formula represents an improved delivery solution to manage insufficient bio-accessibility of quercetin and enhance the bioavailability of the compound. For the Quercetin LipoMicel formulation, a liquid micelle matrix composed of natural food-grade ingredients was developed to facilitate bioavailability. The purpose of the present study, then, was to evaluate solubility and gastrointestinal absorption of quercetin in this novel delivery system in healthy adults.

\section{Material \& Methods}

\section{Formulation AO (free Quercetin); AP (Quercetin Phytosome); AW (Quercetin LipoMicel)}

Three different preparations of quercetin were procured from commercial sources. Each product provided in this study was intended to provide $500 \mathrm{mg}$ of "free" quercetin. Quercetin LipoMicel ${ }^{\circledR}$ (batch no.: 869911) was prepared and provided by Natural Factors (Coquitlam, BC, Canada). Quercetin Phytosome ${ }^{\circledast}$ hard capsules (lot no.: 404996) were manufactured and purchased from Thorne Research Inc. (Thorne, USA). Free quercetin formulated in hard capsules (lot no.: IAY-20022B01) were manufactured and purchased from Amazing Nutrition (New Jersey, USA).

Quality control of the quercetin products used in this study was performed before testing-such as appearance, average mass, uniformity of mass, HPLCmeasured content of quercetin, and microbiological quality was tested.

The first formula is provided as a single hard capsule containing quercetin dihydrate, cellulose, magnesium stearate, and silicon dioxide. For the second product, Quercetin Phytosome two hard capsules containing $250 \mathrm{mg}$ each of Quercetin Phytosome including 
leucine, microcrystalline cellulose, hypromellose capsule, silicon dioxide were tested. The third formulation, Quercetin LipoMicel formula, is composed of quercetin encapsulated with medium chain triglycerides. Its micellular membrane contained food-grade excipients (patent pending). $250 \mathrm{mg}$ of Quercetin LipoMicel was formulated into soft-gel capsules containing gelatin, glycerin, purified water, carob powder, medium chain triglycerides, Stevia rebaudiana leaf, peppermint essence, and phosphatidylcholine (lecithin). Soft gels deliver drugs in solution, suspension or paste form and yet offer solid dosage form [14]. The hydrophobic active ingredient is dissolved in a hydrophilic solvent, and, when crushed or chewed, the solution is directly released into gastric juice. This facilitates a faster absorption from the gastrointestinal tract into the blood streamresulting in higher bioavailability and rapid onset of desired therapeutic effects. Also, soft gels have been reported to improve the extent of absorption as well as to significantly reduce plasma variability. Preparations of liquid-filled soft gel have further proven beneficial to oxidative or hydrolytic degradable drugs $[14,15]$.

\section{Solubility Study}

Individual quercetin formulations used in this study were investigated in terms of their solubility in water and simulated gastrointestinal media. 1-2 g of each of the quercetin samples was added to $10 \mathrm{~mL}$ of simulated media resulting in a total concentration of $500 \mathrm{mg}$ quercetin. The gastrointestinal media were prepared according to the method published by the USP. Briefly, for preparing the simulated gastric fluid ( $\mathrm{pH} 1.2), 2 \mathrm{~g}$ of sodium chloride (Sigma) and $3.2 \mathrm{~g}$ of purified pep$\sin$ (VWR) were dissolved in $7 \mathrm{~mL}$ of hydrochloric acid (Sigma) and made up to $1000 \mathrm{~mL}$ with distilled water.

The simulated intestinal fluid was prepared by dissolving $6.8 \mathrm{~g}$ of monobasic potassium phosphate (Sigma) in $250 \mathrm{~mL}$ distilled water, mixed and then $77 \mathrm{~mL}$ of $0.2 \mathrm{~N}$ sodium hydroxide (Sigma) and $500 \mathrm{~mL}$ of distilled water were added. Next, $10 \mathrm{~g}$ of pancreatin (porcine, Sigma) was added and the media adjusted to pH 6.8 with sodium hydroxide or hydrochloric acid and diluted to $1000 \mathrm{~mL}$.

For the determination of solubility in water, samples were vortexed briefly to suspend visible particles, and then sonicated for 15 minutes at $37^{\circ} \mathrm{C}$ before filtering through $0.45 \mu \mathrm{m}$ polytetrafluoroethylene (PTFE) filters (Chromatographic Specialties, Canada) into glass vials for analysis.

For solubility in simulated gastric and intestinal fluid, samples were vortexed briefly to suspend visible particles, and subsequently sonicated for 60 minutes at $37^{\circ} \mathrm{C}$ before filtering through $0.45 \mu \mathrm{m}$ PTFE filters into glass vials for analysis. Filtered samples contained only particles smaller than $0.45 \mu \mathrm{m}$. Filtered samples were analyzed using a Thermo Ultimate 3000 RS UHPLC system with a quaternary pump delivering a binary gradient of $0.2 \%$ phosphoric acid (HPLC grade, VWR) in HPLC grade water (Fisher Scientific) and HPLC grade acetonitrile (Fisher Scientific) through an Agilent Poroshell EC-C18 $100 \times 2.1 \mathrm{~mm}, 2.7 \mu \mathrm{m}$ column at $0.400 \mathrm{~mL} / \mathrm{min}$. Gradient was linearly increased from $10 \%$ acetonitrile to $100 \%$ acetonitrile over a period of 5 minutes. The column was equilibrated with the starting conditions for 3.3 minutes before next injection. Column oven was set to $40^{\circ} \mathrm{C}$ and data collected at $375 \mathrm{~nm}$. Particle size of the different quercetin formulations in water was measured by means of a Malvern Mastersizer 3000 Laser Diffraction Particle-size analyzer.

\section{Morphological Evaluation}

Atomic force microscopy (AFM) image analysis was performed to gain topographical information of Quercetin LipoMicel. ICSPI nGauge AFM (ICSPI Corp., Canada) revealed nanoscale insights and a three-dimensional surface profile using micro-electro-mechanical systems technology (Figure 1).

\section{Subjects and Study Design}

Twelve healthy volunteers participated in an open, crossover bioavailability study performed with three different commercial quercetin products. Study participants were of both sexes and aged between 21-65 (5 male and 7 female) - with BMIs under 30. Participants were non-smokers, were not taking any prescribed

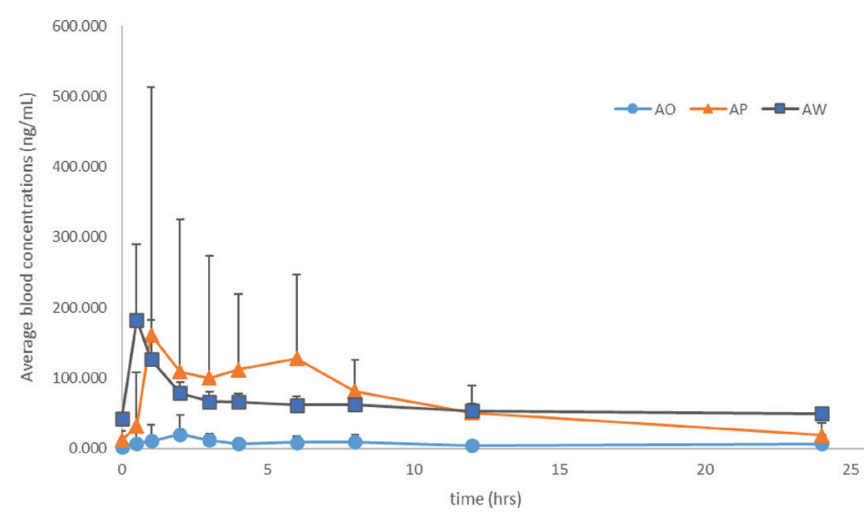

Figure 1. Pharmacokinetic profile of quercetin in study participants. Plasma concentrations after the oral administration of quercetin formulated in different treatments at a total dose of $500 \mathrm{mg}$. AO (free quercetin), AP (Quercetin Phytosome), AW (Quercetin LipoMicel). Values are means \pm SD, $n=12$ for each point. AW significantly different from $A O$ treatment, $P<0.005$ performed with ANOVA followed by post hoc $t$ tests (Bonferroni Correction). 
medication and were not allergic to the treatment products or the provided food.

Each treatment was administered in a total dose of $500 \mathrm{mg}$ quercetin to the participants. The treatments included one hard capsule of $500 \mathrm{mg}$ free quercetin, two gelatin capsules of $250 \mathrm{mg}$ Quercetin Phytosome Complex, or two soft-gelatin capsules of $250 \mathrm{mg}$ Quercetin LipoMicel. The washout period between the treatments was one week. In contrast to hard capsules consisting of "two-pieces", a soft gel capsule represents a one-piece/unitary package containing a liquid, a suspension, or a semisolid-imbedded between gelatin outer layers $[14,15]$. Such soft gel delivery systems have many advantages over other traditional dosage forms e.g., tablets and/or hard capsules, as aforementioned.

Twenty-four hours before each treatment, participants were instructed to avoid consuming quercetin-containing food such as capers, onions, green tea or fruits like apples, berries, grapes, or any supplements containing quercetin. Each participant acted as their own control. On the day of the trials, participants were asked to arrive after an overnight fast. Time zero blood samples were taken upon arrival. Next, each participant received their treatment of quercetin gelatin capsules (one of the three samples), and treatments were consumed with a glass of water along with a standardized breakfast consisting of one hardboiled egg with ham and cheese served in a commercial brioche bun. Capillary whole blood was collected at time points $0.5,1,2,3,4,6,8,10,12$, 24 hours. A standardized lunch consisting of tofu soup and rice along with an optional milk tea was served after the 4-hour blood sample. Dinners were not standardized; participants could consume any meal that does not contain foods as listed above. Coffee, juice, or water could be consumed in any volumes and at any time during the course of the study. However, other than the optional milk tea, no other tea-based beverage was consumed.

At each blood sampling time point, participants first washed their hands thoroughly with soap and warm water. A study assistant wiped the finger to be pricked for blood with a disposable alcohol wipe and lanced the participant's finger with a single-use lancet. The first drop of blood was wiped off with a sterile cotton gauze, and $50 \mu \mathrm{L}$ of blood were collected into pre-labelled microcentrifuge tubes mixed with $5 \mu \mathrm{L}$ of $10 \% \mathrm{w} / \mathrm{w}$ ascorbic acid in water. Samples that were not processed the same day were kept frozen at $-20^{\circ} \mathrm{C}$ until further processing and analysis. Processed samples were analyzed by LC-MS within 24 hours after processing.

\section{Ethical Considerations}

In accordance with the Declaration of Helsinki, written informed consent was obtained from all participants involved in the study. The volunteers were assured that declining to participate in the study or leaving the study at any point would not affect their relationship with ISURA.

\section{Sample Preparation and Analysis}

All samples collected during the multiple product tests were immediately frozen as whole blood. Blood samples from the same week are batched together and run as a sequence on LC-MS. For sample preparation, whole blood samples were first thawed at room temperature and then $50 \mu \mathrm{L}$ of blood were treated with $100 \mu \mathrm{L}$ of $\beta$-glucuronidase (from Helix pomatia, Millipore-Sigma, $\geq 100,000$ IU diluted to $330 \mathrm{IU}$ ) in pH 5 buffer and incubated for one hour at $37^{\circ} \mathrm{C}$. (-)-Epicatechin (MilliporeSigma) was included as internal standard before sample analysis. $400 \mu \mathrm{L}$ of ethanol (99\%, Commercial Alcohol, Canada) was added to extract the samples. Samples were sonicated while maintained in a water bath at room temperature for 15 minutes. After extraction, tubes were centrifuged at $16,000 \times \mathrm{G}$ for 5 minutes at $25^{\circ} \mathrm{C}$. The supernatant was transferred onto a microplate for LC-HRMS analysis. Processed samples were analyzed using a Thermo Vanquish UHPLC system coupled to a Thermo QExactive Orbitrap Mass Spectrometer. Briefly, liquid chromatography was carried out with a binary solvent gradient progressing from $10 \% \mathrm{~A}$ to $100 \% \mathrm{~B}$ in 5 minutes and equilibrated for 3.5 minutes before the next injection. The mobile phases were $0.5 \%$ formic acid in water in A and $0.5 \%$ formic acid in acetonitrile in B. A Phenomenex Kinetex PS C18, $100 \mathrm{~mm} \times 2.1 \mathrm{~mm}$, $2.6 \mu \mathrm{m}$ column was used to perform the separation at a flow rate of $400 \mu \mathrm{L} / \mathrm{min}$. LC-MS grade solvents and formic acid are obtained from Fisher Scientific (Canada).

The Orbitrap mass spectrometer was calibrated at 70,000 resolution with an accepted range for mass deviation of +/- 5.0 ppm using Thermo Pierce LTQ Velos ESI Positive Ion Calibration Solution and Thermo Pierce ESI Negative Ion Calibration Solution. To reduce interference with sample matrix, the mass spectrometer was operated in Parallel Reaction Monitoring (PRM) Mode with heated electrospray at a resolution of 35,000 and isolation window of $1.0 \mathrm{~m} / \mathrm{z}$. Epicatechin (internal standard) was detected as a hydrogen adduct with a product ion of 139.0388. Quercetin was detected as a hydrogen adduct with product ions of 153.0182, 229.0495, 257.0442, 165.0183, and 137.0235.

Data were collected using Thermo Xcalibur 5.0 and analyzed with Thermo TraceFinder 5.0 software with default mass tolerance set to $5.00 \mathrm{ppm}$. Concentrations of quercetin in capillary whole blood were determined based on internal standard calibration with a 6-point calibration curve using quercetin dihydrate as the chemical standard (Millipore Sigma). 


\section{Statistical Analysis}

Calculated concentrations were recorded with Microsoft Excel and calculated on a per individual basis using each participant as his/her own control, using aggregated averages. Results of the pharmacokinetic study are reported as difference of means $\pm \mathrm{SD}(n=12)$ as a method of converting all variance of the individual values to a similar scale. Statistical data analysis was performed by ANOVA followed by post hoc $t$ tests (Bonferroni Correction). Data were considered significant at $P<0.05$.

\section{Results}

\section{Solubility}

Results of the in-vitro solubility studies performed with different formulations of quercetin are summarized in Table 1 . Considering the solubility in gastric conditions ( $\mathrm{pH}$ 1.2), LipoMicel had the highest stability-protecting quercetin against the premature degradation in the stomach. Intestinal solubility of quercetin ( $\mathrm{pH}$ 6.8) could be substantially improved by using new technologies such as LipoMicel or Phytosome formulations. This may correlate with a smaller particle size of quercetin aggregates when encapsulated into carriers as compared to free quercetin. Conversely, free quercetin's poor solubility in water and gastric media seemed to be associated with its large aggregate particle size as demonstrated in Table 1.

\section{Pharmacokinetic Properties}

Three commercially available quercetin products were evaluated pharmacokinetically in vivo. The efficacy of the LipoMicel delivery form on the bioavailability of quercetin in the body was compared to that of free quercetin and the phytosome formulation. Plasma concentrations after the oral administration of a total dose of $500 \mathrm{mg}$ quercetin formulated in the three different products were measured.

No withdrawal by any subject occurred during the course of the trial.

The quercetin plasma concentrations plotted against time are displayed in Figure 1 for each of the individual preparations. As illustrated, a significantly higher blood concentration $(\mathrm{ng} / \mathrm{mL})$ of quercetin was achieved when administered by the LipoMicel formulation as compared to free quercetin. The LipoMicel formulation attained a peak blood concentration of quercetin $\left(\mathrm{C}_{\max }: 182.85 \mathrm{ng} / \mathrm{mL}\right)$ within the shortest period of time $\left(\mathrm{T}_{\max }: 0.5 \mathrm{~h}\right)$. Table 2 presents the individual pharmacokinetic parameters-such as $\mathrm{C}_{\max }, \mathrm{T}_{\max }$ and $t_{1 / 2}$-obtained with the different dosage forms. 8- and 9-fold increases in AUC and $\mathrm{C}_{\max }$ were attained with LipoMicel in contrast to free quercetin; 10-fold higher quercetin blood concentrations were still measured after 12 hours. The LipoMicel formulation achieved an increase of roughly $18 \%$ in $\mathrm{C}_{\max }$ including a 2 -fold reduction in $\mathrm{T}_{\max }$-when compared to Quercetin Phytosome. The AUC for Quercetin Phytosome was slightly higher than for Quercetin LipoMicel (1536.00 +/- $115.66 \mathrm{ng} \mathrm{h/mL}$ vs 1477.27 +/- 25.57), but longer steady state levels of quercetin were provided by LipoMicel.

\section{Morphological Evaluation}

A 3-dimensional reconstruction of the surface of the LipoMicel formulation was created using nGauge AFM. AFM image analysis revealed nanoscale insights of LipoMicel showing quercetin dispersed in microdroplets with a distinct spherical shape (Figure 2).

\section{Safety}

No adverse events or GI intolerances were reported throughout the trial.

\section{Discussion}

A major factor limiting the clinical application of quercetin is its poor pharmacokinetic profile. Therefore, various galenic formulations have been designed in an effort to improve quercetin's rate of absorption.

In the present study, a formulation designed and manufactured by Natural Factors was found to significantly increase the in vivo bioavailability of quercetin. By encapsulating quercetin within a liquid micelle matrix, the (LipoMicel) delivery system was associated with enhanced permeability and retention effects-with elevated blood concentrations of quercetin.

It has been reported that quercetin formulated into a lecithin-based Phytosome delivery system could substantially improve its solubility and bioavailability in humans [16]. In the case of the LipoMicel technique, a

Table 1. Solubility and particle size of different quercetin formulations.

\begin{tabular}{lcccc}
\hline Formulation & Water $(\mathrm{mg} / \mathrm{mL})$ & Simulated gastric $(\mathrm{mg} / \mathrm{mL})$ & Simulated intestinal $(\mathrm{mg} / \mathrm{mL})$ & Particle size $(\mu \mathrm{m})$ \\
\hline Free Quercetin & 1.17 & 0.477 & 0.086 & 217 \\
Quercetin LipoMicel & 0.034 & 0.001 & 1.768 & 110 \\
Quercetin Phytosome & 8.217 & 10.788 & 10.925 & 20.3 \\
\hline
\end{tabular}

Note: Quercetin tested in water, simulated gastric fluid pH 1.2; and simulated intestinal fluid pH 6.8. Particle size measured in water. 
Table 2. Pharmacokinetic parameters obtained after oral administration of different quercetin formulations.

\begin{tabular}{lcccc}
\hline Formulation & $\mathrm{AUC}_{0-24}(\mathrm{ng} \mathrm{h} / \mathrm{mL})$ & $\mathrm{C}_{\max }(\mathrm{ng} / \mathrm{mL})$ & $\mathrm{T}_{\max }$ (hours) & $\mathrm{t}_{1 / 2}$ (hours) \\
\hline AO (free Quercetin) & $172.87 \pm 12.21$ & $19.77 \pm 27.29$ & $2 \pm 0.15$ & $11.66 \pm 0.55$ \\
AW (Quercetin LipoMicel) & $1477.27 \pm 25.57 *$ & $182.85 \pm 106.64^{*}$ & $0.5 \pm 0.02^{*}$ & $8.29 \pm 0.49 *$ \\
AP (Quercetin Phytosome) & $1536.00 \pm 115.66$ & $150.27 \pm 61.43$ & $1 \pm 0.30$ & $6.02 \pm 0.52$ \\
\hline
\end{tabular}

Note: Results are expressed as the mean \pm standard deviation; $n=12$. One-way ANOVA with post hoc $t$-tests (Bonferroni Correction). ${ }^{*} P<0.005$ in comparison to treatment $\mathrm{AO}$.

Abbreviations: $C_{\max }$ maximum plasma concentration; $A \cup C$ area under the concentration-time curve; $t_{1 / 2}$ elimination half-life.
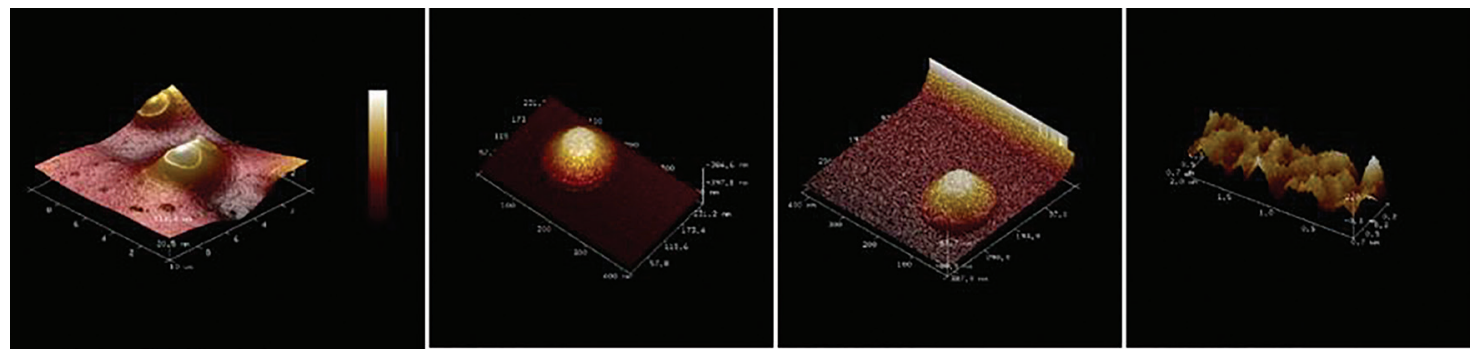

Figure 2. Topography AFM images of Quercetin LipoMicel formulation in 3-dimensional representation.

liquid micelle matrix that disperses quercetin into tiny micro-droplets (Figure 1) resulted in greater bioaccessability and absorption of the compound (Table 1). Oral administration of LipoMicel allowed for better gastric stability and intestinal solubility of quercetin compared to free quercetin. The LipoMicel formulation was associated with a significant improvement of AUC, $\mathrm{C}_{\max }$ and $\mathrm{T}_{\max }$-tested at a standardized dose of $500 \mathrm{mg}$ nonmodified quercetin. It is noteworthy that approximately, 8- and 9-fold increases in AUC and $\mathrm{C}_{\max }$ were attained when using LipoMicel in contrast to free quercetin. 10-fold higher quercetin blood concentrations were measured after 12 hours. Comparing the two formulations-Quercetin Phytosome and Quercetin LipoMicel, an increase of roughly $18 \%$ in $\mathrm{C}_{\max }$ including a 2 -fold reduction in $\mathrm{T}_{\max }$ was accomplished by the LipoMicel carrier design. Both products appeared to enhance bioavailability more than unmodified quercetin. Unlike the other treatments, the LipoMicel formula reached a peak blood concentration of quercetin within a minimum time of 30 mins. Over the total measurement period-significant higher, stable blood concentrations of quercetin were observed with LipoMicel in relation to free quercetin. Similarly, the use of LipoMicel resulted in improved gastric stability and intestinal solubility of quercetin compared to free quercetin when tested in simulated gastrointestinal conditions. This is supported by the early rise of quercetin in vivo-with peak blood concentrations at $0.5 \mathrm{~h}$-as well as with sustained high concentrations throughout the $24 \mathrm{~h}$ study period. Delivery vehicles such as LipoMicel or Phytosome that encapsulate quercetin at an aggregate particle size of $\leq 110 \mu \mathrm{m}$ were associated with greater intestinal solubility, as suggested in in vitro experiments. Conversely, a larger aggregate particle size such as that of free quercetin was associated with poor solubility in water and in simulated static gastrointestinal conditions.

Both delivery systems, LipoMicel and Phytosome, appeared to be proficient carriers for enhancing the in-vivo absorption of quercetin. While the Phytosome formulation appears to improve quercetin bioavailability by increasing its solubility in water, the LipoMicel formulation demonstrates an alternate route to increased bioavailability: one that may not be related to solubility. One possible explanation for the greater bioavailability of Quercetin LipoMicel could involve bypass of efflux by P-glycoprotein (P-gp) transport protein, which is extensively present along the intestinal tract $[17,18]$. However, to evaluate this possibility, further experiments perhaps with caco-2-cell models might be helpful to gain insight into mechanisms of intestinal permeability and stability. Furthermore, long-term in vivo studies with quercetin on a larger sample size are required in order to obtain more comprehensive pharmacokinetic data. Also, the effect of different food substrates on quercetin absorption, including differences between fasting and various standardized meals should be studied in future investigation.

\section{Conflict of Interest}

JS, CC, KR, MD, and YCK are employees of Isura and have no conflicts. $\mathrm{MH}$ and $\mathrm{ML}$ receive consulting fees from the Factors Group of Companies. RG is the owner of the Factors Group of Companies. Isura is a not-forprofit independent organization. 


\section{Authors' Contributions}

JS, CC, KR, MD, YCK: contributed equally to this work.

JS: helped to design research, analyzed and interpreted data and drafted the original manuscript.

(D) https://orcid.org/0000-0003-2784-1435

CC: designed and supervised research and contributed to drafting the manuscript—original draft preparation.

$K R, M D, Y C K$ : conducted experiments and contributed to drafting the manuscript—review and editing.

$\mathrm{MH}, \mathrm{ML}, \mathrm{RG}$ : contributed to drafting the manuscriptreview and editing.

All authors gave final approval of the version to be published.

\section{Funding}

The study was funded by Natural Factors.

\section{References}

[1] Anand David AV, Arulmoli R, Parasuraman S. Overviews of biological importance of quercetin: A bioactive flavonoid. Pharmacogn Rev 2016;10:84-9. http://doi. org/10.4103/0973-7847.194044

[2] Derosa G, Maffioli P, D'Angelo A, Di Pierro F. A role for quercetin in coronavirus disease 2019 (COVID-19). Phytother Res 2020. https://doi.org/10.1002/ptr.6887

[3] Wang W, Sun C, Mao L, Ma P, Liu F, Yang J, Gao Y. The biological activities, chemical stability, metabolism and delivery systems of quercetin: A review. Trends in Food Science \& Technology. 2016;56:21-38. https://doi. org/10.1016/j.tifs.2016.07.004

[4] Batiha GE-S, Beshbishy AM, Ikram M, Mulla ZS, El-Hack MEA, Taha $A E$, et al. The pharmacological activity, biochemical properties, and pharmacokinetics of the major natural polyphenolic flavonoid: Quercetin. Foods 2020. https://doi.org/10.3390/foods9030374

[5] Mckee DL, Sternberg A, Stange U, Laufer S, Naujokat C. Candidate drugs against SARS-CoV-2 and COVID19. Pharmacol Res 2020;157:104859. https://doi. org/10.1016/j.phrs.2020.104859

[6] Kinker B. Quercetin: A promising treatment for the common cold. J Anc Dis Prev Rem 2014. http://doi. org/10.4172/2329-8731.1000111

[7] Solnier J, Fladerer J-P. Flavonoids: A complementary approach to conventional therapy of COVID-19? Phytochem Rev 2020:1-23. https://doi.org/10.1007/ s11101-020-09720-6

[8] Yuan K, Zhu Q, Lu Q, Jiang H, Zhu M, Li X, et al. Quercetin alleviates rheumatoid arthritis by inhibiting neutrophil inflammatory activities. J Nutr Biochem 2020;84:108454. https://doi.org/10.1016/j.jnutbio.2020.108454

[9] Manach C, Scalbert A, Morand C, Rémésy C, Jiménez L. Polyphenols: Food sources and bioavailability. Am J Clin Nutr 2004;79:727-47. https://doi.org/10.1093/ ajcn/79.5.727

[10] Mullen W, Edwards CA, Crozier A. Absorption, excretion and metabolite profiling of methyl-, glucuronyl-, glucosyl- and sulpho-conjugates of quercetin in human plasma and urine after ingestion of onions. Br J Nutr 2006;96:107-16. https://doi.org/10.1079/bjn20061809

[11] Németh K, Plumb GW, Berrin J-G, Juge N, Jacob R, Naim $H Y$, et al. Deglycosylation by small intestinal epithelial cell beta-glucosidases is a critical step in the absorption and metabolism of dietary flavonoid glycosides in humans. Eur J Nutr 2003;42:29-42. https://doi.org/10.1007/ s00394-003-0397-3

[12] D'Archivio M, Filesi C, Varì R, Scazzocchio B, Masella R. Bioavailability of the polyphenols: Status and controversies. Int J Mol Sci 2010;11:1321-42. https://doi. org/10.3390/ijms11041321

[13] Đorđević V, Balanč B, Belščak-Cvitanović A, Lević S, Trifković K, Kalušević A, et al. Trends in encapsulation technologies for delivery of food bioactive compounds. Food Eng Rev 2015;7:452-90. https://doi.org/10.1007/ s12393-014-9106-7

[14] Prasad VD. Formulation and modifying drug release from hard and soft gelatin capsules for oral drug delivery. IJRDPL 2017;06:2663-77. https://doi.org/10.21276/ IJRDPL.2278-0238.2017.6(4).2663-2677

[15] Damian F, Harati M, Schwartzenhauer J, van Cauwenberghe $\mathrm{O}$, Wettig SD. Challenges of dissolution methods development for soft gelatin capsules. Pharmaceutics 2021. https://doi.org/10.3390/ pharmaceutics13020214

[16] Riva A, Ronchi M, Petrangolini G, Bosisio S, Allegrini P. Improved oral absorption of quercetin from quercetin phytosome $^{\circledR}$, a new delivery system based on food grade lecithin. Eur J Drug Metab Pharmacokinet 2019;44:169-77. https://doi.org/10.1007/s13318-0180517-3

[17] Wang S-Y, Duan K-M, Li Y, Mei Y, Sheng H, Liu H, et al. Effect of quercetin on P-glycoprotein transport ability in Chinese healthy subjects. Eur J Clin Nutr 2013;67: 390-4. https://doi.org/10.1038/ejcn.2013.5

[18] Kaur V, Garg T, Rath G, Goyal AK. Therapeutic potential of nanocarrier for overcoming to P-glycoprotein. J Drug Target 2014;22:859-70. https://doi.org/10.3109/10611 $86 \times .2014 .947295$ 


\section{Article Information}

Managing Editor: Kieran Cooley

Peer Reviewers: Olina Dagher, Kent TJ Chen

Article Dates: Received Apr 20 21; Accepted Jun 29 21; Published Jul 2621

\section{Citation}

Please cite this article as follows:

Solnier J, Chang C, Roh K, Du M, Kuo YC, Hardy M, Lyon M, Gahler R. Quercetin LipoMicel—A Novel Delivery System to Enhance Bioavailability of Quercetin. Journal of Natural Health Product Research. 2021 Jul 26: 3(2). https://jnhpresearch.com/index.php/jnhpr/article/view/17

DOI Link: https://doi.org/10.33211/jnhpr.17

\section{Copyright}

(c) Julia Solnier, Chuck Chang, Kyle Roh, Min Du, Yun Chai Kuo, Mary Hardy, Michael Lyon, Roland Gahler (2021). Published first in the Journal of Natural Health Product Research. This is an open access article distributed under the terms of the Creative Commons Attribution License (https://creativecommons.org/licenses/by/4.0/), which permits unrestricted use, distribution, and reproduction in any medium, provided the original work, first published in the Journal of Natural Health Product Research, an NHP Publications journal, is properly cited. The complete bibliographic information, a link to the original publication on https://www.jnhpresearch.com, as well as this copyright and license information must be included.

Journal of Natural Health Product Research NHTPTURTIMATIONS

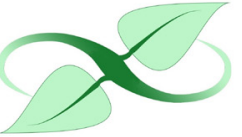

Canadà

Does the safety, efficacy, and quality of natural health products matter to YOU? Submit your research article to the Journal of Natural Health Product Research!

Pre-submission inquiries? Send us an email at editorial.office@jnhpresearch.com Facebook, Twitter and LinkedIn: @NHPPublications 